\title{
Web annotation technologies for learning
}

John Casey

City of Glasgow College

\section{Introduction}

This introduces web annotation tools and standards and their potential to support learning and teaching across a range of activities using existing web resources, with an initial discussion of implications for academic practice. It concludes with a short description of current web annotation tools for text, images and audio-visual resources.

\section{What is web annotation?}

To start with, it helps to remind us of traditional uses of annotation in the pre-digital world of paper. These have included 'marginalia' - i.e. handwritten notes in the margins of books, underscores and highlights etc. - used by individuals. More wide-ranging applications of annotation in paper media have included scholarly works, such as annotated anthologies of poetry or annotated bibliographies of quantum physics. From this perspective, web-based annotation is an attempt to recreate and extend that functionality as a new layer on top of the web. This brings all the affordances of the web environment; annotations can be 'published', shared, managed and curated, as well as re-published via social media channels.

Annotations can be linked, shared between services, tracked back to their origins, searched and discovered and stored wherever the author wishes. Web-based annotations enable a reader to interact with web pages to create her/his own content linked back to the original web page and these annotations (and their connections to the original web page) can, in turn, be annotated by others.

\section{Applications for Learning}

With web annotation, what is developing are powerful ways of interacting with web content and other web users. These tools bring some great opportunities for education and research. In education, the use case is especially compelling: students have been writing in their books since the book was invented. As books, texts, images and videos studied in schools and college migrate online, these marginal notes can become media-rich and shared with others. In face-to-face, online and blended classrooms, collaborative annotation combines traditional literacy with more emergent understandings of the types of skills students need to develop to be successful in digital environments.

At the I Annotate 2016 conference in Berlin (http://iannotate.org/2016/) a range of educational examples of web annotation were provided including the Lacuna project from Stanford University (http://bit.ly/2nFmBE7) and the PAHSIT project at University of Colorado Denver (http://bit.ly/2EB8BD2). What was striking was that the annotation technologies were enabling the kinds of threaded discussion that we normally associate with forums in Virtual Learning Environments (VLEs). However, in this case, the discussion was happening in the 'annotation layer' centred on shared web resources; the W3C infographic below (Figure 1.) 
will help with visualising this, as will the screenshot of the 'Compass' journal article with an annotation (Figure 2.).

\section{Implications for academic practice}

A good, single sentence, description of the potential user experience of web annotation is provided by a leading scholarly annotation service:

"A conversation layer over the entire web that works everywhere, without needing implementation by any underlying site." (Hypothes.is: https://web.hypothes.is/about/)

This conveys perhaps the most important thing about web annotations - they are independent of the original web page, exist separately from it and are under the control of the annotator. Yet, to a third-party viewer of the annotations, they co-exist with the original content, because this annotation layer is under the control of the annotator and not the original web page author/publisher. Thus, annotations can circumvent decisions that content owners have made about whether they want 'commenting' in the first place. In academia and elsewhere, this raises important questions about the traditional role of the author, copyright, reputation and ownership.

\section{Technical Architecture of Web Annotations}

The W3C (World Wide Web Consortium) is establishing data standards to provide a good foundation for future developments in the key areas of inter-operability, preservation and sustainability. The pages of the W3C web annotation working group

(https://www.w3.org/annotation/) provide a good source of information; the illustration (and the accompanying text below) is taken from there and provides a useful visualisation of how web annotation works in practice (W3C, 2017).

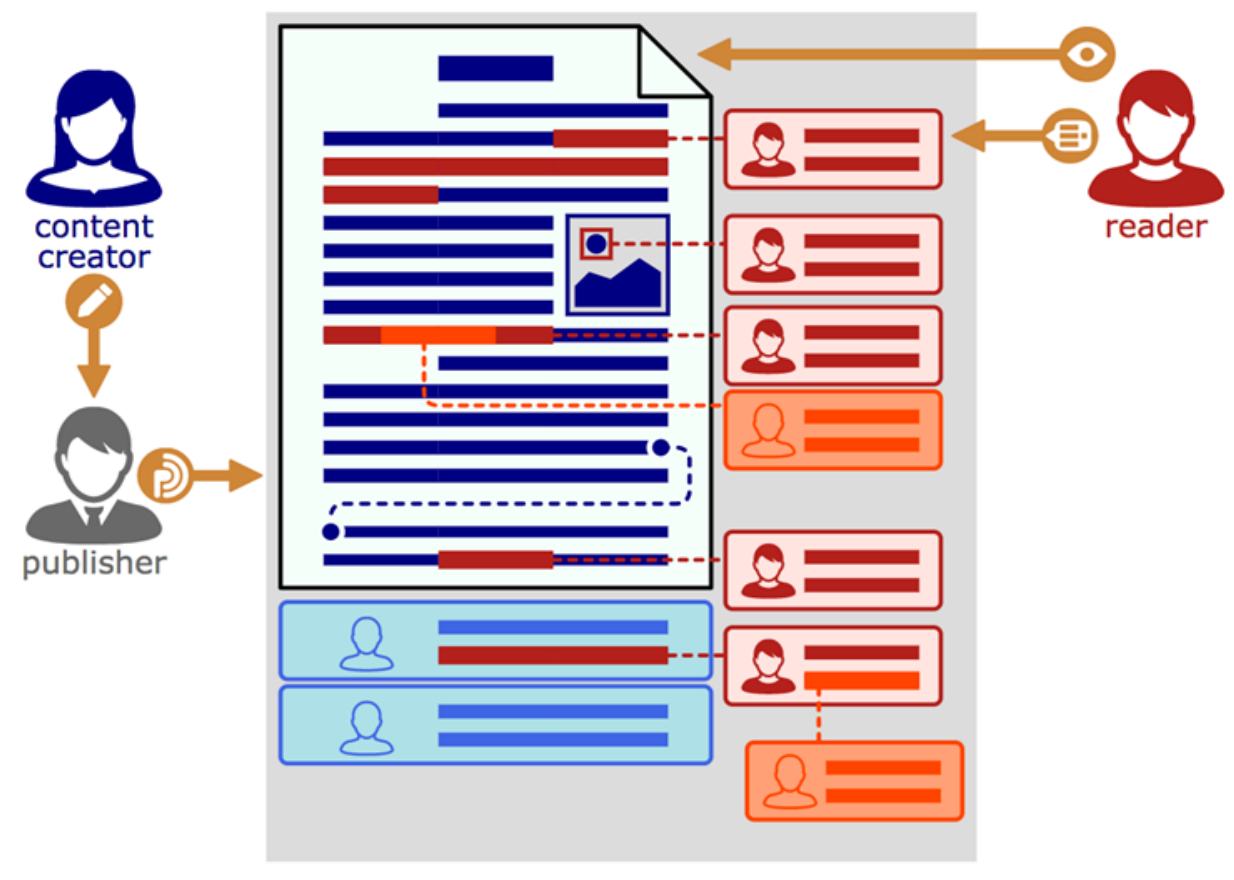

Figure 1: A generic web annotation architecture, showing a reader creating captions (righthand column) in relation to selections on a web page 
"With annotations, a reader can target a discrete selection. A selection can be text, a section of an image, a location on an interactive map, a timestamp of a video or audio, or a data representation that has an underlying data source. $A$ reader can even annotate a footnote ... or a traditional site comment. Other readers can annotate sections of content that overlap existing annotations ... and even annotate annotations themselves, in threaded replies. "

Copyright Statement (C (23 February 2017) World Wide Web Consortium, (MIT, ERCIM, Keio). http://www.w3.org/Consortium/Legal/2015/doc-license

\section{Web Annotation Tools}

Annotating textual resources on the web is relatively well advanced, with perhaps the best example being the open source Hypothes.is project (https://web.hypothes.is) that provides a free subscription service for users. An example of a Hypothes.is service user's public annotation of a Compass journal article page is given below in a screen shot featuring a single annotation; the public annotation is also available at this web link: http://bit.ly/2EatgA4.

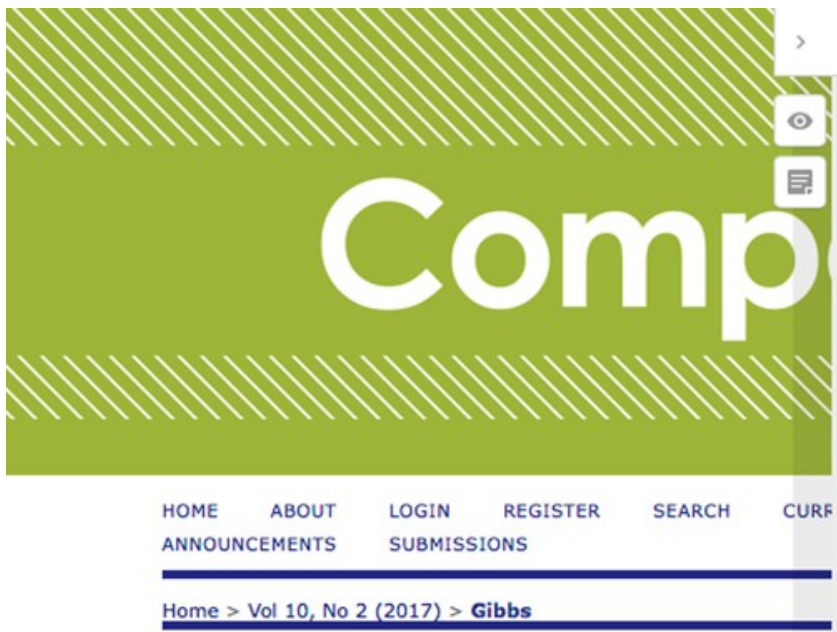

Evidence does not support the rat
TEF Graham Gibbs

Abstract

The Teaching Excellence Framework (TEF) has evolved since it was fi guidance to institutions on its implementation reveals a number of si evidence, common sense and fairness. Institutions may well implem mechanisms in response, as they have always done regardless of th assurance demands. However the rationale of the TEF remains - and rationale that this paper focuses on. It is argued here that its interp educational quality, employability and value for money ratings, 1 and are not supported by evidence. Making fine judgments about .... between institutions. Some of its proposed metrics are invalid. Its be of experts to make sound auality judged is attexts to make sound quality judgments is not well founded give attempts to make such judgments about teaching quality in higher the TEF intends. The TEF seems unlikely to be perceived, by most, a:
(3) Public

$Q \uparrow_{\downarrow}<$ Sign up / Log in.
To annotate this document create a free account or log in

\section{Show all annotations}

john_casey

The Teaching Excellence Framework (TEF) has evolved since it was first announced, and HEFCE guidance to institufil ... More

This is interesting I can annotate this abstract in html format but I cannot annotate the article in the pop up window because it is in PDF format. So going forwards it might be a good idea to have a html format for the articles? Discuss... PDF HTML
This is a public annotation created with Hypothesis. To reply or make your own annotations on this document, create a free account or $\underline{\log }$ in.

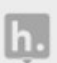

Figure 2: A Compass article page, with annotation available at: http://bit.ly/2EatgA4 
Annotation of images is developing rapidly, with work being led by the IIIAF consortium (http://iiif.io). An example of image annotation is given by the work of Digirati with the Wellcome Trust and their development of a Universal Player for the trust (http://bit.ly/2s6785s), with the planned addition of annotations.

Annotation of audio-visual resources on the web is more emergent. In this connection, the work of the Clipper (Clipper 2017) project, funded by Jisc (http://bit.ly/1SizMCd), is providing some useful insights, making it easier for learners and teachers to analyse, manage and collaborate using online audio and video resources. 